\title{
The Descriptivist vs. Anti-descriptivist Semantics Debate between Syntax and Semantics
}

\author{
Enrico Cipriani \\ University of Torino
}

\begin{abstract}
In this paper, I will focus on the debate between descriptivism and anti-descriptivism theory about proper names. In the introduction, I will propose an historical reconstruction of the debate, and focus in particular on Russell and Kripke's treatments of proper names. Strong criticisms will be advanced against Kripke's hypothesis of rigid-designator and, more clearly, against the consequent distinction between the epistemic and metaphysical level that Kripke proposes to explain identity assertions between proper names. Furthermore, I will argue, that, pace Kripke, Russellian treatment of proper names allows to capture all our semantic intuitions, and also those semantic interpretations which concern context-belief sentences. I will close the introduction by focusing on a criticism that Kripke rightly points out against an example that Russell proposes in his On Denoting. Section 2 will be devoted to Russellian solution: I will show that not only Russell's logical treatment of proper names allows to answer to Kripke's criticism to Russell's example, but also that such treatment can disambiguate and express all our semantic intuitions about Frege's puzzle sentence "Hesperus is Phosphorus." I will then show that, contrarily, Quinian solution (discussed in section 3) and Kripkian one (see section 4) are not satisfactory to capture our semantic knowledge about Frege's sentence. Furthermore, in section 5, I will focus on Kripke's distinction between epistemic and metaphysical level to deal with identity assertions between proper names, and I will logically show that such distinction is not plausible. In section 5, then, I will show that Russellian solution allows to explain context-belief sentences, contrarily to what Kripke thinks. In Conclusions, I will summarize what I have argued in the text.
\end{abstract}

Keywords: rigid-designator, epistemic-metaphysical levels, syntax-semantics interface, descriptivism vs. anti-descriptivism

\section{Introduction: How to Pass from Syntax to Semantics}

The debate between descriptivist and anti-descriptivist rages in philosophy of language, since when Kripke proposed his extraordinary theory of rigid-designator. The authors engaged in such debate challenge by very complex and articulated arguments, and a lot of volumes should be written to summarize what was happening. The casus belli is represented by the following question: Does at least one definite description correspond to a proper name? Are proper names individual constants which denote the same object in all possible worlds or are they definite descriptions?

The problem of how to logically interpret proper names is due to Frege, who thought that proper names

Enrico Cipriani, Ph.D. student, Department of Philosophy, University of Torino, Italy; main research field: Philosophy of Language-Epistemology of Linguistics. 
were disguised definite descriptions: The name "Aristotle," for example, corresponds to something like "Plato's pupil." Russell was of the same opinion, as he explicitly stated in The Philosophy of Logical Atomism (1918-1919, 200-1).

Althought both Frege and Russell had proposed such hypothesis about proper names, the most famous theory is Searle's (1958) one, which supported the idea that grammatical proper names correspond to disjunctions of definite descriptions.

However, it was Wittgenstein (1953) to advance a skeptical doubt about such proposal, when, in the Philosophical Investigations, he wrote:

When I make a statement about Moses, am I always ready to substitute some one of these descriptions for "Moses"? I shall perhaps say: By "Moses" I understand the man who did what the Bible relates of Moses, or at any rate a good deal of it. But how much? Have I decided how much must be proved false for me to give up my proposition as false? Has the name "Moses" got a fixed and unequivocal use for me in all possible cases? Is it not the case that I have, so to speak, a whole series of props in readiness, and am ready to lean on one if another should be taken from under me and vice versa? (37)

As Casalegno wrote:

Even if Wittgenstein prefers to accumulate questions instead of proposing a thesis, what he wants to suggest is clear. We often are able to, or, better: We think that we are able to describe the reference of a proper name in different ways-when this happens, nothing authorizes us to favor a specific description and to say that the name is synonymous with such description. Each possible way to identify the reference of a name is relevant, and at the same time no one is decisive. $(1997,230)$ (my translation)

The strongest criticisms to Russellian hypothesis about proper names, however, came from Kripke, who, since seventies $(1971 ; 1980)$, argued that proper names do not correspond to definite descriptions, but to rigid-designator, namely to individual constants which denote the same object in all possible worlds. The name "Aristotle" refers to the same object whatever is the world that we consider, contrarily to the definite description "Alexander the Great's master." Consequently, when we talk about counterfactual situations in which Aristotle is a carpenter, we talk about the same object $x$ denoted in our world by "Aristotle."

Kripke's theory allows to solve some relevant problems before unsolvable, and to avoid contradictions. For example, if "Aristotle" corresponds to "Alexander the Great's master," so both "Aristotle was Aristotle" and "Aristotle was the Alexander the Great's master" must be analytic: a very counter-intuitive conclusion, to which both Frege-Russell and Searle's theory lead.

If it is true that Kripkian proposal allows to explain a lot of problematic cases, on the other hand, it leads to different perplexities. The most striking case is that which concerns identity assertions between proper names. According to Kripke, both "Hesperus is Hesperus" and "Hesperus is Phosphorus" are necessary truth; it is however unquestionable that few people would be disposed to say that the latter sentence is necessary, namely that it is true in every possible world. To face such possible criticism, Kripke distinguished between epistemic and metaphysical dimension, namely between a priori and a posteriori knowledge and necessary and contingent truth: "Hesperus is Hesperus" is necessary a priori, while "Hesperus is Phosphorus" is necessary a posteriori. Such a distinction is not so clear, and leads to different problems (see section 4). Another important problem which arises from Kripke's theory is presented by Kripke himself, in a famous article of 1979, "A Puzzle about Belief," where the author shows that if we accept the hypothesis that proper names correspond to rigid-designators and if we admit two intuitive principles (the disquotational principle and the translation principle), we see that in epistemic (belief) sentences we have unsolvable contradictions. A lot of pages have 
been written on Kripke's puzzle, and different proposals have been advanced. What is clear is, anyway, that this puzzle is a puzzle only in Kripkian perspective tout court.

We can react in two ways in front of Kripke's puzzle: We can reject the hypothesis of proper names as rigid-designators, by getting however to the contradictions which characterize descriptive approach to proper names, or we can try to reinterpret some of the fundamental assumptions of Kripke's theory, to understand which historical factors have determined the birth of such theory. I will not begin such enterprise, too complex and hard for me, but I will limit to two observations. The first one concerns the definite descriptions that we associate to proper names, and the observation is the following: The fact that no rigid definite description corresponds to proper names does not mean — nor entail — that no contingent definite descriptions are associated to proper names. Kripke's puzzle is burn on a misunderstanding, which can be expressed in this way: The individual constant denotes surely the same object in all possible worlds, and the same can be true for two constants which denote the same object $x$; however, to say that does not mean that, when she uses the proper name, the speaker does not associate to the proper name some definite descriptions. Contrarily, it seems implausible to think that we cannot have knowledge by acquaintance of things without having a minimal knowledge by description. There can be cases where the speakers associate to different proper names which have the same reference different psychological contents, as it is in the case of a speaker that, without having any information about references of two names $n$ and $m$, nevertheless she associates to the names different mental contents. More easily, if someone talks to me about a person whose name is Jones, and she gives me a description of Jones, I will use "Jones" as a rigid-designator, but I will not denote directly Jones, but an object which is created on the basis of the description of Jones that I have. The same is true in the case of fictional objects: The name "Sherlock Holmes" needs at least a description to be used; nevertheless, it is used as a rigid-designator. In other words, it is useful to distinguish between the modality by which a reference is denoted, and the use of the proper name (as rigid-designator or definite description).

The second observation concerns the modality of denotation. The hypothesis that grammatical proper names correspond to logical constants characterizes Russell's thought: Russell distinguished between "Scott is a man" and "the author of the Waverley is a man," by arguing that while the former is expressed by the proposition " $x$ is a man," this is not true for the latter. He wrote:

According to the view that I advocate, a denoting phrase is essentially part of a sentence, and does not, like most single words, have any significance on its own account. If I say "Scott was a man", that is a statement of the form "x was a man" and it has "Scott" for its subject. But if I say "the author of the Waverley was a man", that is not a statement of the form "x was a man", and does not have "the author of the Waverley" for its subject. (Russell 1905, 488)

In this way, Russell has the possibility to solve the problem connected with truth values for sentences which contain phrase which does not denote anything. It must be reminded that Russell draws this distinction — which a purely syntactic distinction — without giving any information about the way the individual constant must be interpreted.

Russell tells us, in other words, that "Scott" corresponds to a singular term expressed by a constant, while it is not so for definite descriptions. He does not tell us, however, that the individual constant $s$ is a constant which denotes directly the object Scott; contrarily, as we have seen, he thinks that proper names correspond to definite descriptions. How can we explain such contradiction, namely the fact that, on the one hand, Russell uses an individual constant to translate grammatical proper names, while on the other he tells us that 
grammatical proper names have a content? The question is even more legitimate if we consider that Russell calls "logical proper names" only those expressions which are called indexicals nowadays, namely those terms which refer directly, by ostension, to the object they denote (Russell 1918-1919, 200-1). The answer can be: the distinction in the translation that proper names and definite descriptions do not correspond to a distinction in the way the speakers use proper names and definite descriptions. This distinction is the result of further and subsequent elaboration and interpretation of Russell's logic, due to Kripke, who, as a supporter of Direct Reference Theory, argues that proper names, as demonstratives, refer directly to the object they denote. However, Russell does not tell us anything of this kind: He tells only that proper names and definite description correspond to two different syntactic structures, and this is all. The reason which is pushed to think that proper names denote directly the object is an historical reason. Model semantics theory has shown that, given a model, the individual constants denote the same object whatever are the assignment functions. Consequently, there are cases where the constant $c$ denotes the same object of variable $x$ and other where it denotes the same object denoted by $y$. The direct consequence in natural language semantics is that a proper name denotes the same object in all possible worlds. This perspective, however, requires God's perspective, or, better, the perspective of the logician who, if she had infinite time, could compute all the identity relations between the individual constant and other logical elements of the model (being understood Quinian skepticism). What has not been understood is that when we reason on how speakers use proper names, translated as individual constants, speakers are the result of an assignment function: They are not, instead, the logicians who observe the model "from the high." This explains our doubt in front of the hypothesis that the sentence "Hesperus is Phosphorus" would be necessary: Kripke tells us that after empirical analysis (being the sentence a posteriori), we can get to this necessary truth; he seems to forget, however, that when they express this judgment, speakers are inside the world, where they have different properties, determined by the assignment function, and cannot be the logician who observes all the worlds from the high.

A stronger criticism can be advanced. Even if we admit that we can establish if "Hesperus" and "Phosphorus" denote the same object $x$, it a traditional problem in philosophy must be faced: What is the object denoted by "Hesperus" and "Phosphorus"? In other words, given a domain D of object which is part of a model, in D we will find Venus, namely we will find a planet with some properties. However, to say that we will find Venus corresponds to say that we will find an object $o$ which is denoted by us, in our world, by "Venus;" the problem is that we do not know what Venus is, nor in which sense the notion of object of domain must be intended: Of a model, we can read only logical relations between constants and variables (and different function of the structure), but we cannot read the relations between logical elements and objects of the domain, because such objects, actually, are not definable. This problem is clear to the logicians, and could be called the ontological problem. The ontology of possible worlds is one of the most discussed arguments in philosophy of language, but it does not seem that a conclusion has been drawn yet.

These observations do not entail that it does not make difference if we choose to translate proper names as constants or definite description: It is evident that if we translate proper names in two different ways, different consequences will concern the relation between logic and semantics. One of these consequences has been pointed out by Kripke, in Russell's Notion of Scope (2005), where Kripke discusses also Russell's sentence about King George curiosity, putting in evidence that if "George IV wishes to know if the author of the Waverley" is interpreted with primary occurrence for the definite description and secondary occurrence for the proper name, George IV is actually interested in the law of identity. Kripke wrote: 
Now, Russell comments, "This would be true, for example, if George IV had seen Scott at a distance, and had asked 'Is that Scott?' (Russell 1905, 52)." Common sense suggests this would be a reasonable interpretation. However, we are not just dealing with common sense here, but with Russell's theory. Russell's characterization is a good common sense interpretation of the large scope analysis. However, this is an existential quantifier and the variable takes a unique value. What can that value be? Scott himself! But then the first gentleman of Europe is interested in an instance of the law of identity, after all! Since the value of the propositional function here is Scott=Scott, the question would express an interest in the law of identity. Now, in the paper, Russell introduces his famous distinction between knowledge by acquaintance and knowledge by description and says we often use definite descriptions to denote objects we know only by description and are not acquainted with. (2005, 1023-4)

What Kripke points out is that if we assume that "Scott" corresponds to a logical proper name (namely, an individual constant which denotes directly the object it refers to), then the sentences express that George IV is interested in knowing if the object Scott is identical to itself (considering that the definite description has primary occurrence). However, Kripke reminds, Russell (1918-1919, 62) adopts the hypothesis that grammatical proper names correspond to logical proper names only when they are used in knowledge by acquaintance contexts, and not when the speaker has knowledge by description of the name. Consequently, George's interest in the law of identity does not hold, since, in the example presented by Russell, George has knowledge by description of the name "Scott" (probably, he associates to the name some definite descriptions), so that the King wants to know if the object the author of the Waverley satisfies the properties associated to the name "Scott." Assuming that this theoretical solution allows to avoid George's interest in the law of identity, nevertheless the distinction between knowledge by acquaintance and knowledge by description entails two different logical treatments of grammatical proper names.

Actually, a point must be stressed here. What Kripke points out is that since the individual constants are not quantified, it is not possible to distinguish between wide and narrow scope for the proper name; but, if we accept the reflections just proposed, Russell seems to think that we can interpret the proper name as confined to King's beliefs and idiolect. If parentheses which close the identity relation express a belief - and, if we pass on a metalinguistic level, the assertion — of George IV, $s$ is interpreted as used by George IV, and not by who utters the sentence. It rests however the impression that Kripke's observations hit the target. That the individual constant is not sufficient to deal with proper names is evident if we consider that a sentence like "George IV wishes to know if Scott is Scott" can mean that George IV wishes to know if Scott (the name "Scott" is de dicto in respect of the epistemic operator) is identical to himself, that of the object denoted de re (see below) by the name "Scott:" the King wants to know if he is identical to himself, or if of the object Scott; the King wants to know if he is or not Scott, in the sense that he wants to know if "Scott" refers to such object. This puzzle will be solved in the following pages. Kripke thinks, anyway, that Russell is conscious of such problem, and that in $O n$ Denoting he distinguishes between knowledge by acquaintance and knowledge by description to face such a problem. If we admit this interpretative proposal of Kripke (which seems to be right), we can say that, according to Russel, we must distinguish between grammatical proper names as individual constants - namely, logical proper name - and grammatical proper name as definite descriptions. As I will argue in the following pages, this solution, contrarily to Quine and Kripke's ones, is the only which allows to capture and describe correctly all—or many of—our semantic intuitions.

\section{Russell's Solution}

If we adopt the proposal to merge together descriptivism and anti-descriptivism, we see that a lot of 
problems which hit both the positions are solved. As we have seen, according to Russell, grammatical proper names can correspond to constants or definite description. To show how Russellian view solves different problems, let's consider first of all the name "Scott." According to Russell (1905), we will say that "Scott" can correspond to $s$ or to a definite description which has the following form:

$$
\exists(\mathrm{x})[\mathrm{S}(\mathrm{x}) \wedge \forall(\mathrm{y})(\mathrm{S}(\mathrm{y}) \rightarrow \mathrm{x}=\mathrm{y})] \equiv \exists !(\mathrm{x})[\mathrm{S}(\mathrm{x})],
$$

where "S" represents the predicate "to be Scott." By this solution, we can solve the ambiguity rightly pointed out by Kripke $(2005,1024-5)$ mentioned above. The sentence "George IV wishes to know if the author of the Waverley is Scott" is translated as:

$$
\exists !(x)[A W(x)] \wedge \text { George IV wishes to know if }(\exists y S(y) \wedge y=x) .
$$

The logical form just proposed means that of the author of Waverley: The King wants to know if he is denoted by "Scott," where "Scott" is expressed by a uniquely existential quantification which contains the predicate "to be Scott," which expresses the semantic content associated to "Scott" by King George. An alternative way to translate the sentence is:

$$
\exists !(\mathrm{x})[\mathrm{AW}(\mathrm{x})] \wedge \text { George IV wishes to know if }(\mathrm{S}(\mathrm{x})) \text {, }
$$

which expresses that of the object the author of Waverley, George IV wishes to know if he is Scott, namely if he satisfies the property to be Scott. As we will see in the last section, by the Russellian distinction, we can explain ambiguities which would be, otherwise, unexplainable.

We can now consider Frege's puzzle. Let's call it E; E corresponds to "Hesperus is Phosphorus."

E has two different (intuitive) interpretations, as in the following:

(a) Venus is Venus;

(b) The entity denoted by (the sense) "Hesperus" and the entity denoted by (the sense) "Phosphorus" are the same object (have the same reference) (or, in Voltolini's (2014) words, they are no other than the same thing).

(a) and (b) are two interpretations that a (good) theory of meaning must be able to express in logical terms. If we adopt Russellian distinction, we see that (a) is expressed by,

$$
(h=p) \text {, }
$$

while (b) corresponds to:

$$
\exists !(x) H x \wedge \exists !(y) P(y) \wedge x=y .
$$

\section{Quine's Solution}

As it is well-known, Quine supports the idea that logical constants have to be avoided in first-order logic, and that each denoting phrase of human language has to correspond to a definite description existentially quantified. Quine $(1953,167, \S 7 ; \S 8)$ proposes this solution, in which E would be translated by:

$$
\exists !(\mathrm{x}) \mathrm{Hx} \wedge \exists !(\mathrm{y}) \mathrm{P}(\mathrm{y}) \wedge \mathrm{x}=\mathrm{y} .
$$

Of course, this solution does not allow to express (a), which is instead a perfect valid interpretation of $\mathrm{E}$.

Different criticisms have been advanced against Quinian hypothesis. The strongest one is that of Hochberg, who, in an article of 1957, pointed out that Quine's proposal entails that clear analytic truths are instead contingent. For example, the sentence "Phosphorus is shining or Phosphorus is not shining" would not be analytic, in Quinian translation. It is surprising to notice that Hochberg's criticism is right, but the example he proposes is wrong — resoundingly wrong. In fact, he says that a sentence of the form ( $\mathrm{P}(\mathrm{a}) \bigvee \neg \mathrm{P}(\mathrm{a})$ ), where "a" is a proper name, is not analytic. For example, "Phosphorus is shining or (Phosphorus) is not shining," that 
Quine would translate as:

$$
\exists !(\mathrm{x})[\mathrm{P}(\mathrm{x}) \wedge \mathrm{S}(\mathrm{x})] \vee \exists !(\mathrm{x})[\mathrm{P}(\mathrm{x}) \wedge \neg \mathrm{S}(\mathrm{x})],
$$

is false if Phosphorus does not exist. Actually, Hochberg does not remember that Russell (1905) shows that the negation operator can have wide or narrow scope in this kind of sentence. In fact, the sentence can be translated by,

$$
\exists !(x)[P(x) \wedge \mathrm{S}(\mathrm{x})] \vee \neg \exists !(\mathrm{x})[\mathrm{P}(\mathrm{x}) \wedge \mathrm{S}(\mathrm{x})],
$$

which is a tautology. As I have said, however, Hochberg's criticism is right. Let's consider the sentence "Phosphorus is Phosphorus." If the proper name is translated by a definite description, then the logical form is:

$$
\exists !(\mathrm{x})[\mathrm{P}(\mathrm{x})] \wedge \mathrm{x}=\mathrm{x},
$$

which is not a tautology, since it is false if Phosphorus does not exist. To avoid this conclusion, we should suppose that the quantifier ranges over a fix domain of the model, and so we should adopt a necessitist hypothesis (Williamson 2013); however, Quine likes desert landscapes.

\section{Kripke's Proposal}

Contrarily to Quine, Kripke thinks that proper names correspond only to rigid-designators (except for some rare cases: see descriptively proper names), so that E expresses, according to Kripke, a necessary truth. The meaning that Kripke attributes to $\mathrm{E}$ is (a), and never (b), which is not contemplated as a possible meaning for E. However, E, contrarily of a sentence like "Hesperus is Hesperus," is necessary a posteriori. I have already mentioned some serious difficulties that we meet when we adopt Kripke's distinction between epistemic and metaphysical levels; as I have said, this distinction requires God's perspective.

Who wants to defend Kripke's solution could again, anyway, argue something like that: It is not necessary a perspective "from the high" to establish that "Hesperus is Phosphorus" is a necessary truth, since in virtue of our knowledge of first-order logic, we know that if in our world $e$ and $f$ are co-designator, we have discovered that they are co-designator in every possible world. Actually, such objection would not be efficacious, not only for the theoretical reasons just pointed out. Actually, the problematic point concerns not only the fact that the denotation on object of the domain is a very vague notion, since relata does not exist, but concerns also the fact that, intuitively, there are worlds where we-who study planets' movements - have a different epistemic condition from that one that we have in our (this) world, so that in some worlds we could discover that actually Hesperus is not Phosphorus. In other words, the metaphysical dimension is connected with the single worlds where speakers are in a specific epistemic condition.

To better understand this problem, let's consider again Kripke's proposal. According to Kripke, if E is true, then $\mathrm{E}$ is necessary. We have:

$$
\mathrm{E} \rightarrow \mathrm{N}^{1} \mathrm{E} .
$$

Let's consider, first of all, that the conditional "if $\mathrm{E}$ is true, then $\mathrm{E}$ is necessary" is true even if the antecedent is false and the consequent is true, and so if it is false that $\mathrm{E}$ but it is necessary that $\mathrm{E}$ : This would be sufficient to reject Kripke's proposal. Let's consider that, if the conditional just mentioned is right, if $\mathrm{E}$ is true then $\mathrm{E}$ is true in every possible world, included a world $\mathrm{W}_{\mathrm{n}}$ where human beings think that is false. It must be noticed that Kripke's solution entails that if $\mathrm{E}$ is false, that it is necessary false. We have so:

$$
\neg \mathrm{E} \rightarrow \mathrm{N} \neg \mathrm{E} \text {. }
$$

Let's imagine that $\mathrm{E}$ is false in $\mathrm{W}_{1}$ : If it is false, then it is false in every possible world; but this way we get to the paradoxical conclusion that in every possible world $\mathrm{E}$ is true and false at the same time, because $\mathrm{E}$ is 
necessarily true and false at the same time; this is a contradiction.

A further criticism in Kripke's hypothesis concerns the fact that we cannot express (b) by it. While (a) should be expressed by:

$$
(h=p) .
$$

(b) would correspond to:

$$
\exists !(\mathrm{x}) \mathrm{H}(\mathrm{x}) \wedge \exists !(\mathrm{y}) \mathrm{P}(\mathrm{y}) \wedge \mathrm{x}=\mathrm{y} .
$$

These two logical forms allow to explain and disambiguate also our judgments about the sentence "necessarily, Hesperus is Phosphorus," which would be true according to Kripke. Any speaker would intuitively say that "necessarily, E" is true if $E$ is read as (a), but false if read as (b). And, in fact,

$$
\mathrm{N}(e=f) \text {, }
$$

is true, while,

$$
\mathrm{N}(\exists !(\mathrm{x}) \mathrm{H}(\mathrm{x}) \wedge \exists !(\mathrm{y}) \mathrm{P}(\mathrm{y}) \wedge \mathrm{x}=\mathrm{y}),
$$

is false in every of the scalar lectures that we can propose.

\section{A Possible Russellian Account of Belief Sentences}

In the introduction, I have reminded that Kripke (1979) concludes that epistemic and belief sentences are too complicate and chaotic to be explained in a rigorous way. Now I want to show, contrarily, that by Russellian treatment of proper names we can solve the ambiguities which characterize such sentences: To do so, I want to mention a couple of examples. In the end of the introduction, I focused on the ambiguity of a sentence like "George IV wishes to know if Scott is Scott." I have said that such sentence can have different interpretations. The most relevant are:

- George IV wishes to know if the object that he knows as Scott is identical to himself;

- Of Scott, George IV wishes to know if he is identical to himself;

- Of Scott (of entity Scott), George IV wishes to know if he has the property to be Scott.

These interpretations can correspond to the situations, respectively, where George IV talks about Scott and asks (for any reason that do not interest us) if Scott is identical to himself, where the King wonders about the fact that the person that we (but not he) call Scott, if he is identical to himself (for example, the King denotes Scott with a different name from "Scott," or he points to him), and, at the end, where George IV asks if the person that we denote by "Scott" has the property to be Scott (for example, the King asks if a specific person that he sees is the person who wrote Waverley and whose name - the King knows to be-is Scott). Of course, these interpretations cannot be captured by Quinian or Kripkian views. Contrarily, if we use both descriptivist and anti-descriptivist view, we can translate the sentences in the following ways:

- George IV wishes to know if $(\exists !(x) S(x) \wedge x=x)$;

- $\exists !(\mathrm{x}) \mathrm{S}(\mathrm{x}) \wedge$ George IV wishes to know if $(\mathrm{x}=\mathrm{x})$ ( $\equiv$ George IV wishes to know if $(\mathrm{s}=\mathrm{s})$ );

- George IV wishes to know if (S(s)).

Let's note that if we adopt Kripke's solution, the only translation of the sentence "George IV wishes to know if Scott is Scott" would be:

- George IV wishes to know if $(\mathrm{s}=\mathrm{s})$,

while Quine would translate it as:

- George IV wishes to know if $(\exists !(x) S(x) \wedge x=x)$.

To come back to E, let's consider the sentence "Jones wishes to know if Hesperus is Phosphorus" (let's 
call it $\left.\mathrm{E}_{1}\right) . \mathrm{E}_{1}$ can mean that Jones wants to know if Venus is Venus, if the object that we call-which is de re-Hesperus corresponds to the object that Jones calls Phosphorus (or to the object which has the property to be Phosphorus) or if the two objects that he (which are de dicto in respect of Jones's idiolect) calls Hesperus and Phosphorus are the same thing. These interpretations are provided by:

- Jones wishes to know if $(\mathrm{e}=\mathrm{f})$;

$\bullet \exists !(x) H(x) \wedge$ Jones wishes to know if $(\exists !(y) P(y) \wedge x=y)$ ( $\equiv$ Jones wishes to know if $(P(e)))$;

- Jones wishes to know if $(\exists !(\mathrm{x}) \mathrm{H}(\mathrm{x}) \wedge \exists !(\mathrm{y}) \mathrm{P}(\mathrm{y}) \wedge \mathrm{x}=\mathrm{y})$;

- $\exists$ ! $(\mathrm{x}) \mathrm{H}(\mathrm{x}) \wedge \exists !(\mathrm{y}) \mathrm{P}(\mathrm{y}) \wedge$ Jones wishes to know if $(\mathrm{x}=\mathrm{y})$.

As Russell, "the ambiguity as between primary and secondary occurrences is hard to avoid in language; but it does not harm if we are on our guards against it. In symbolic logic it is of course easily avoided" (1905, 489).

\section{Conclusions}

I have focused on the debate between descriptivist and anti-descriptivist approach to proper names. The origins of the debate have been mentioned in the introduction, where I have focused also on the relation between Russellian treatment of proper names and the implications that such syntactic treatment had in semantics. I have pointed out two observations: The fact that (at least) a definite description is associated to a proper name does not entail that it is necessary to take a descriptivist position about proper names; and Kripke's perspective, which distinguishes between an epistemic and a metaphysical level to explain that identity assertions between proper names, if true, are necessary would require an epistemic condition which does not characterize speakers in their own possible worlds. This last point has been developed in section 4, where I have shown that Kripke's solution leads to contradiction.

In section 2, I have shown Russellian treatment of proper names, and I have pointed out that we must translate grammatical proper names as both individual constants and definite descriptions if we want to face Kripke's (2005, 1023-4) criticisms to Russell's (1905) example. Furthermore, I have examined the sentence "Hesperus is Phosphorus," and I have shown that (at least) two of the most relevant interpretations of such sentence can be captured and expressed only by Russellian solution, while both Quinian and Kripkian ones are not sufficient for this aim.

The moral of the story is very simple: It is not possible to deny that both Quine and Kripke's solutions about proper names allow to solve ambiguities and different (specific) problems that opposing theories cannot solve. However, in the moment when we look at our language using and we want a semantic theory which is able to express all our semantic intuitions (and this would be the principal aim of a semantic theory), we must recognize that both descriptivist and anti-descriptivist approaches, if considered in isolation, fail.

\section{Notes}

1. I use the symbol $\mathrm{N}$ for the modal operator of necessity.

\section{Works Cited}

Casalegno, Paolo. Filosofia del linguaggio (Philosophy of Language). Roma: Nuova Italia Scientifica, 1997. 
Hochberg, Herbert. “On Pegasizing.” Philosophical and Phenomenological Research 17.14 (1957): 551-4.

Kripke, Saul Aaron. "Identity and Necessity.” Ed. Munitz M. K. Identity and Individuation. New York: New York University Press, 1971.

---. “A Puzzle about Belief.” Ed. A. Margalit. Meaning and Use. Dordrecht: Reidel, 1979. 239-83.

---. Naming and Necessity. Oxford: Basil Blackwell, 1980.

---. "Russell's Notion of Scope." Mind 114.456 (2005): 1005-37.

Quine, Willard Van Orman. From a Logical Point of View. Harvard: Harvard University Press, 1953.

Russell, Bertrand. "On Denoting.” Mind 14.56 (1905): 479-93.

---. "The Philosophy of Logical Atomism.” The Monist 28 (1918): 495-527; 29 (1919): 32-63; 190-222; 343-80.

Searle, John Rogers. "Proper Names.” Mind 67 (1958): 166-73.

Voltolini, Alberto. "Contingent Sameness and Necessary Identity.” Ed. Adriano Palma. Castañeda and His Guises: Essay on the Work of Hector-Neri Castañeda. Boston-Berlin: The Gruyter, 2014. 187-206.

Williamson, Timothy. Modal Logic as Metaphysics. Oxford: Oxford University Press, 2013.

Wittgenstein Ludwig. Philosophische Untersuchungen (Philosophical Investigations). Eds. G. E. M. Ascombe and R. Rhees. Oxford: Blackwell, 1953. 